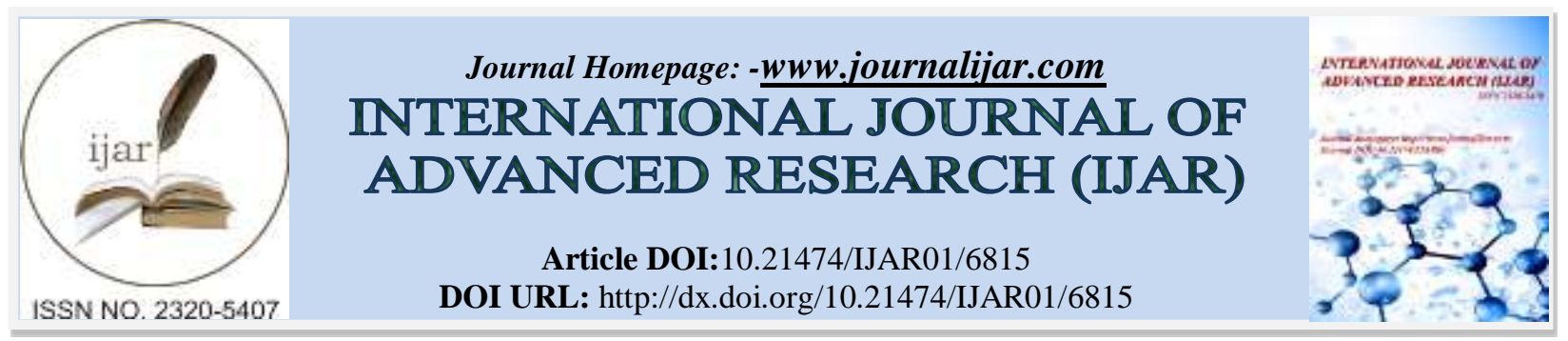

RESEARCH ARTICLE

\title{
ROBOTIC ARM WITH REAL-TIME IMAGE PROCESSING USING RASPBERRY PI, BOTH AUTOMATED AND MANUALLY.
}

\section{Dr. A. Brintha Therese ${ }^{1}$ and Prashant Gupta ${ }^{2}$.}

1. Professor, Department of Electronics \& Communication Engineering, VIT University Chennai, Tamil Nadu, India.

2. B. Tech student, Department of Electronics \& Communication Engineering, VIT University Chennai, Tamil Nadu, India.

\section{Manuscript Info}

\section{Manuscript History}

Received: 22 January 2018

Final Accepted: 24 February 2018

Published: March 2018

\section{Keywords:-}

Robotic Arm, Color detection(RGB),

Raspberry Pi, Python, Automated.

\section{Abstract}

This paper proposes to create a Robotic Arm with Real-Time Image Processing using Raspberry Pi which can either be automated or can be operated manually. In the present era, we are making a robot capable of detecting and placing the pre-specified object. The code for detection of color has been written in Python. For a hardware implementation, we are using raspberry pi which has Raspbian OS based on Debian which is Linux OS.

The robotic arm detects the pre-specified objects and segregates them based on color (RGB). The program includes controlling the robotic arm, capturing the object image processing, identifying the RGB object, using a local page to control motor manually and perform all task automatically using Raspberry Pi.

Copy Right, IJAR, 2018,. All rights reserved.

\section{Introduction:-}

In today's scenario, the robot with high accuracy, high output, and no error is in demand, the precise work or repetitive work is better done with robots, for the robot the sensor or camera is common sense for the machine-like image processing to detect and identify an object and its characteristic which helps to perform a required task.

A Robotic arm can decide the object based on color like Red, Green, and Blue using camera and image processing in raspberry pi.

The main aim of the project is to make a robot that has the capability of pick a pre-specified object and placing it in separate divisions based on color. Raspberry Pi has found its way in many useful and changeable implementations in robotic systems. Raspberry Pi does not implement any usual motor control peripherals and it is available at low cost. The python code has been formulated for creating a robotic arm with image processing and local web page with slider to adjust servo motor position manually.

For the industrial purpose, the robotic arm can help to separate and segregate the object based on color with a good frequency. 


\section{Raspberry Pi (Model B+):-}

Raspberry Pi (model B+) has consisted of an ARM 1176JZF-S processor, 512MB SDRAM shared with GPU, one video and audio output, 4 USB port, one HDMI output. a Video Core IV GPU, $1100 \mathrm{M}$ bit/s Ethernet port, which runs at $700 \mathrm{MHz}$ clock speed. It was developed by a foundation of Raspberry Pi in the UK for the use of computer science education. The third version model B+ of the Raspberry Pi is used in this project. It has 26 pins including 8 General purpose Input/output (GPIO), one SPI bus, one I2C bus, one UART bus and 3.3V, GND and 5V. The Raspberry Pi needs an external Secure Digital(SD) card to store its operating system and also all the user data.

\section{Implementation:-}

Raspbian OS based on Linux is used in raspberry pi for processing the hardware. To identification and detection of the object and its color, the code is written in python. Simple CV libraries have allowed to enhance or process the image called image processing. Which required to identifying the color of an object and control the robotic arm for pick and place pre-specified object operations. The code has local web page also so that using a slider to control the position of the robotic arm.

The implementation process of the proposed work is done in three steps.

1. Image processing techniques, in order to identify the object color(RGB).

2. Assembling and Controlling of Robotic Arm.

3. Integrate the vision system with a Robotic arm to control for pick and place pre-specified object.

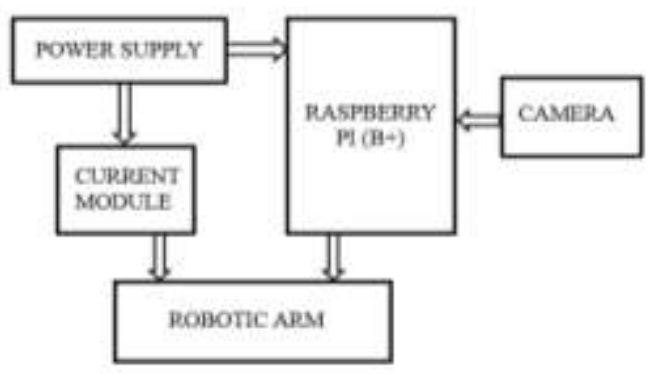

Figure 1:- Proposed System

\section{Image processing techniques, in order to identify the object color(RGB):-}

The value stored for each pixel in the image depends on the color space and color model being used. The color model describes how colors are represented by a set of numbers, where each number corresponds to a different color 'channel'. The color space is the mapping of the channels of the color model to absolute reference values. For example, the widely used color spaces RGB is based on an RGB color model, where each pixel has values in the Red, Green and Blue color channels. Each value represents the intensity of that color relative to the absolute reference colors defined by the RGB color space. The aim is applying a Color Key to an image, picking out a specific color or range of colors in the image and removing them, or replace them with a separate background image. 


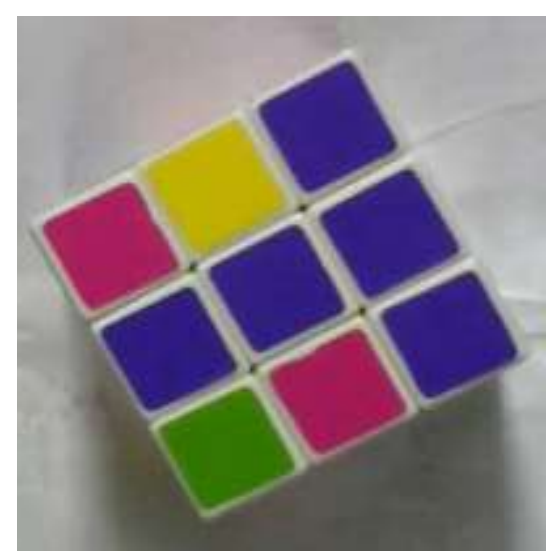

Figure 2:- Real time Image.

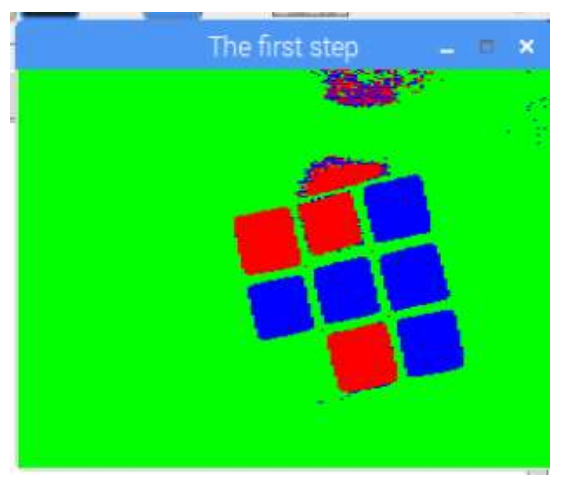

Figure 3:- Color detection.

\section{Assembling and Controlling of Robotic Arm:- \\ Robotic Arm:-}

A mechanical structure with electric motors and end effectors. Look like the human hand to grip the object and place it in the predefined position. The robotic arm attempt to reproduce movement similar to a human arm. It has the base to rotate the arm from 0 degrees to 180 degrees. Like the human hand, arm consists shoulder, elbow, wrist and mechanical gripper to hold the object. The arm can be made up of plastics-based 3D printing, wood, scrap materials, acrylic sheet, Aluminum and more. The arm using in our project has a low-cost arm that has a good quality acrylic sheet. A term that's used for a mechanical system which is the number of independent parameters that define its configuration called DOF (degrees of freedom). when a robot arm is designed the DOF is considered and it is related to roll, yaw, and pitch. The arm has 7 DOF to perform pick and place operation with maximum accuracy. 
Maximum lift: $200 \mathrm{~g}$.

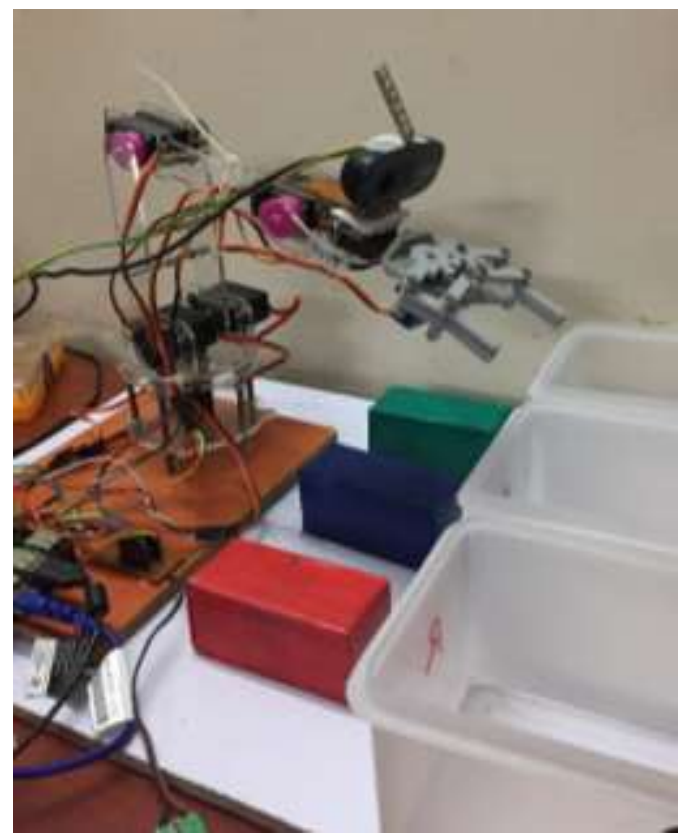

Figure 4:- Designed Robotic arm.

Dimensions: 9" L* 8" $\mathrm{W}^{*} 15$ " $\mathrm{H}$

Weight: $550 \mathrm{~g}$

\section{Power Supply:-}

The raspberry pi is low power controller which uses a micro USB connection to power itself. The recommended amount of current is between 700mA for a Raspberry Pi Model A, and up to 2.5A for a Raspberry Pi 3 Model B+.

\section{USB webcam:-}

In this project, we are using the USB webcam to monitoring the real-time object image on the Raspberry Pi. It's able to deliver a clear 3-megapixel resolution image or 720p HD video recording at 30 frames/sec. The quality and frames/sec of the image helps to enhance and process the image to detect the color of the object with maximum accuracy.

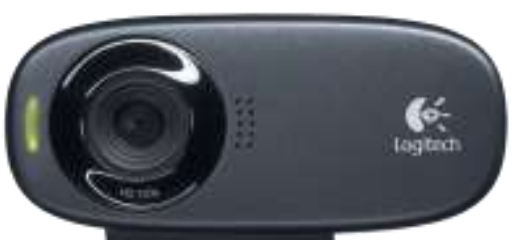

Figure 5:- 720p Logitech webcam.

\section{Current Module:-}

This module allows to maintain the current of a servo motor and work effectively. This robotic arm consists 7 servo motors, and module helps to maintain high speed. A continuous power supply for all servo motors helps to reduce the jerks or choppy movements in them.

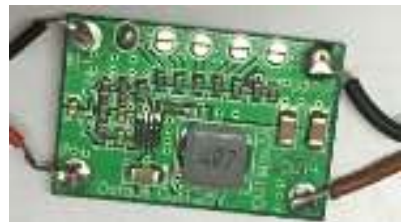

Figure 6:- Current Module 5V, 3A 
Integrate the vision system with a Robotic arm to control for pick and place the desired object:-

The robotic arm can operate as automatic and manual to pick and place the object. The arm and camera are integrated into python code. A local server page has created for manual operation with slider to change the position of the servo motor for undefined instructions. This help for industrial purpose when some correction is required to change the fine position. Python code has instruction for three colors i.e. Red, Green, and Blue to identify the object color and instruct the robotic arm to pick and place pre-specified object. The servo motors can produce the back electromagnetic force(emf) with a high spike of voltage which can damage the controller. In order to save it, either servo motor drivers are used or just diode to stop back emf, because one servo can produce back emf of $100 \mu \mathrm{V}$ and it can multiply by 7 number of servos, but this back emf can stop by using the diode.

Flow Chart:- Automatic

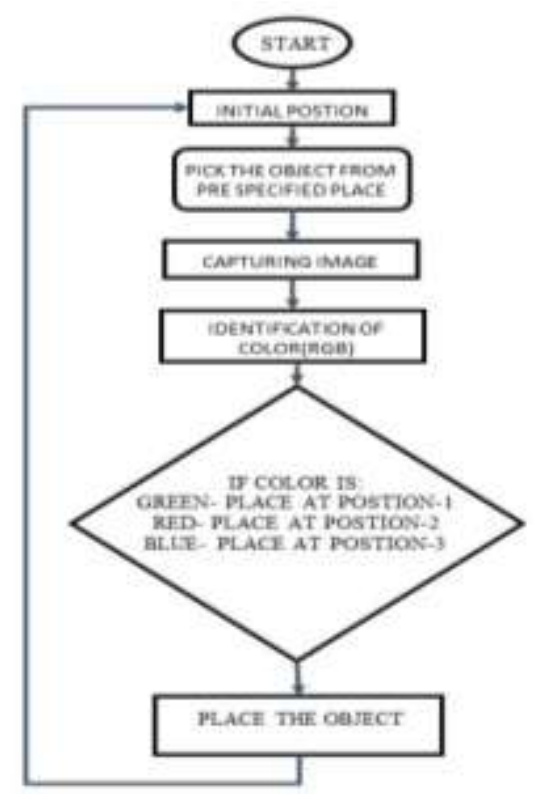

Figure 7:- Flow chart for autonomous operation.

\section{Experimental Results:-}

Lots of experiments have been conducted to control the robot arm using Raspberry Pi. The image has processed to identify the color of object and command send to robotic arm to place the detected object. The robotic arm can have operated in both ways automatically as well as manually which is required for industrial purposes. The experiments have done to acquired maximum accuracy to perform a task. Below figure shows the snapshots of the Object detection and identification of color and manual local web page. The robotic arm can one by one pick the object and detect the object color and placed at the specified place for particular color. 


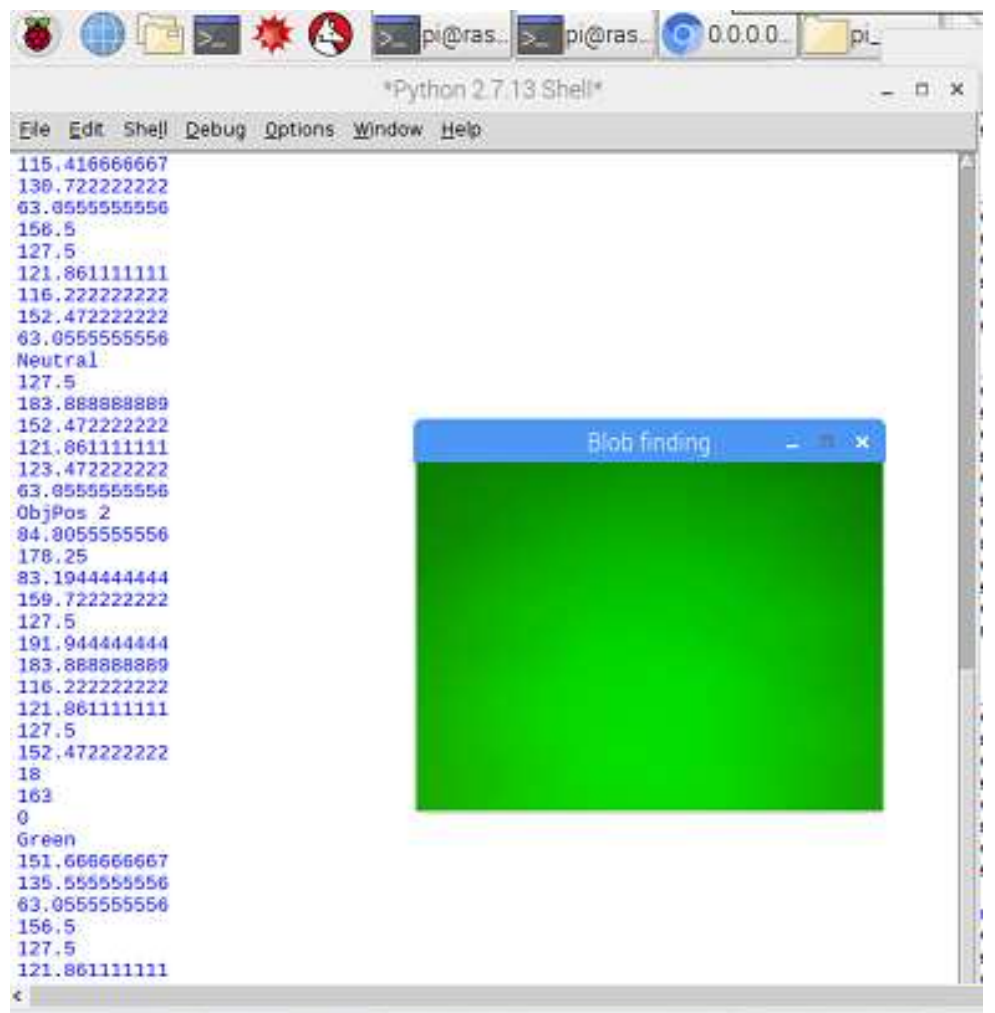

Figure 8:- Robotic arm positions and color detect.

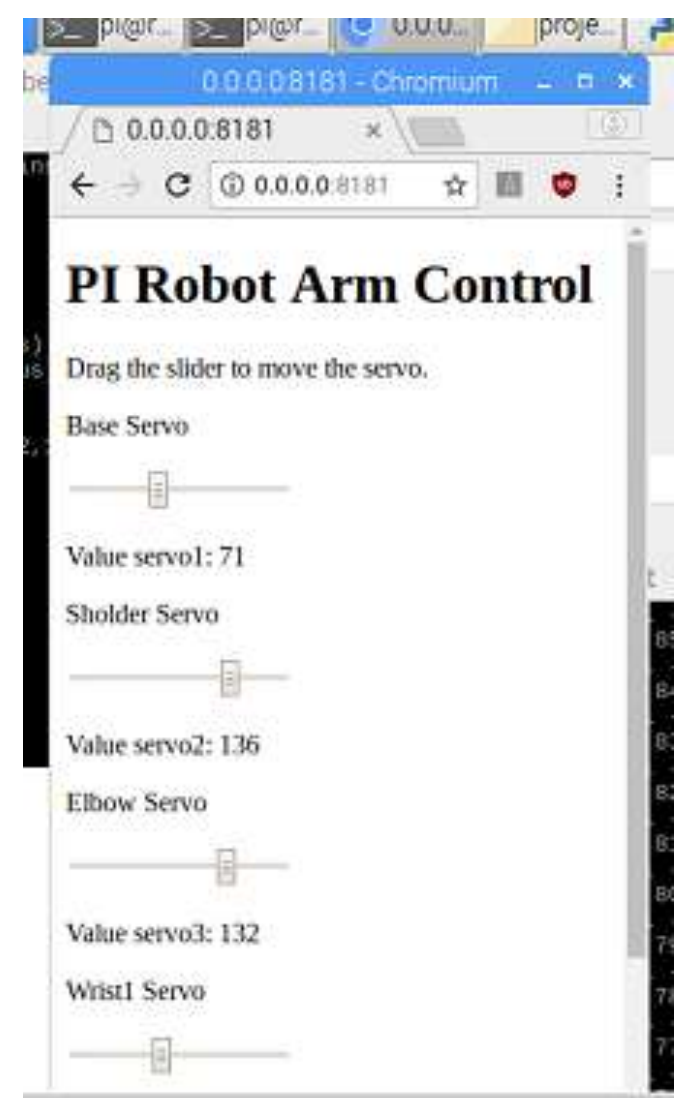

Figure 9:-Web Page for manually control Robotic arm. 


\section{Conclusion:-}

This proposed solution gives better results when compared to the earlier existing systems such as efficient image capture, etc. Identification of colored object by the robotic arm can be controlled both automatically and manually for industrial purpose. The arm can segregate among three colors and place it accordingly. The hardware implementation was carried out using the raspberry pi.

\section{References:-}

1. Dragusu.M," Practical applications for robotic arm by using image processing" system theory, control and computing (ICSTCC), 2012 16th international conference.

2. V.Pereira,"Low-cost object sorting robotic arm using raspberry pi" Global Humanitarian Technology Conference-South Asia Satellite(GHTC-SAS),2014 IEEE.

3. C.Theiss.Steinhagen and I.Iossifidis,"Image processing methods for interactive robot control", proceedings 10th IEEE International Workshop on a robot and human interactive communication,2001.

4. Vijayalaxmi, K.Anjali, B.Srujana, P.Rohith Kumar "Object Detection and Tracking using Image Processing" Electronics and Communications Global Journal of Advanced Engineering Technologies, Special Issue (CTCNSF-2014).

5. R.Szabo,"Automated colored object sorting applications for robot arms" Electronics and Telecommunication (ISETC), 2012 10th International Symposium.

6. P.Ashish and Charu Bhartiya, "Image Processing Based Rose Harvesting System using Raspberry Pi" Electronics International Journal of Engineering Research \& Technology (IJERT)Vol. 4 Issue 04, April-2015.

7. AartiS.Hajari and SaakshiA.shankarpurkar. "Personal Computer-Based Robotic Arm with Vision" International Journal of Image Processing and Vision Sciences(IJIPVS) VOL-1ISS-3,4,2012.

8. Melvin Abraham C, R.Jegan and Shobhitha Ann JOB "OWI535EDGE Robotic Arm Control ElectroMyoGram (EMG)Signals" International Journal of Innovative Technology and Exploring Engineering(IJITEE)VOL-2, ISS-6, may 2013. 